\title{
Classification of Cardiac Arrhythmia based on Hybrid System
}

\author{
T. M. Nazmy \\ Prof. Faculty of computer \\ and information sciences, \\ Ain Shams University,Cairo,Egypt
}

\author{
H. EL-Messiry \\ Faculty of computer \\ and information sciences, \\ Ain Shams University,Cairo,Egypt
}

\author{
B. AL-Bokhity \\ Faculty of computer \\ and information sciences, \\ Ain Shams University,Cairo,Egypt
}

\begin{abstract}
This paper, describes an Intelligent Diagnosis System using Hybrid approach of Adaptive Neuro-Fuzzy Inference System (ANFIS) model for classification of Electrocardiogram (ECG) signals, and comparison this Technique with Feed-Forward Neural Network (FFNN), and Fuzzy Inference Systems (FIS). Feature extraction using Independent Component Analysis (ICA) and power spectrum, together with the RR interval then serve as input feature vector, this feature were used as input of FFNN, FIS, and ANFIS classifiers. six types of ECG signals they are Normal Sinus Rhythm (NSR), Premature Ventricular Contraction (PVC), Atrial Premature Contraction (APC), Ventricular Tachycardia(VT), Ventricular Fibrillation (VF) and Supraventricular Tachycardia (SVT). The results indicate a high level of efficient, the proposed method outperforms the other methods with an impressive accuracy of $97.1 \%$, As for other methods FFNN, FIS results were respectively $94.3 \%, 95.7 \%$.
\end{abstract}

Keywords: ANFIS, adaptive neuro, fuzzy inference system, ECG, ICA, Power Spectral, RR-interval.

\section{INTRODUCTION}

For several years, the automatic classification of ECG signals has received great attention from the biomedical engineering community. This is mainly due to the fact that ECG provides cardiologists with useful information about the rhythm and functioning of the heart. Therefore, its analysis represents an efficient way to detect and treat different kinds of cardiac diseases. A standard scalar ECG is consists of P-wave, PR-interval, PRsegment, QRS complex, ST-segment, and T-wave. The QRS complex is a very important signal that is useful in the diagnosis of Arrhythmias diseases. In this paper, we use the QRS complex to achieve ECG beat classification [1],[2]. In general, the normal ECG rhythm means that there is a regular rhythm and waveform. However, the ECG rhythm of the patient with arrhythmia will not be regular in certain QRS complex [3].

Several researchers have addressed the problem of automatic detection and classification of cardiac rhythms, such as: In [4] used the blind source separation techniques for feature extraction, the features were classified by distance classifier, Bayes Minimum error classifier, and K-Nearest Neighbour classifier. In [5] used Wavelet Transform for ECG feature extraction, using Fuzzy classifier. In [6] used Fuzzy Hybrid Neural Network composed of two sub-networks connected the Fuzzy SelfOrganizing layer performing the pre-classification task and the following Multilayer Perceptron (MLP) working as the final classifier. In [7] used largest Lyapunov exponent, spectral entropy and Poincare geometry for ECG feature extraction, Adaptive Neuro-Fuzzy Inference System (ANFIS) was presented for classification of the ECG signals. In [8] used Wavelet Transform for ECG feature extraction, There are two classifiers that used
Analytical classifier and ANFIS classifier. In [9] used Lyapunov exponents for ECG feature extraction, Adaptive Neuro-Fuzzy Inference System (ANFIS) were presented for classification of the ECG signals. In [10] used three different techniques to extract features from ECG signal which are Fast Fourier Transform, Autoregressive Modeling, and Principal Component Analysis. The features were be classified by using three different classifiers which are ANN, and two types of statistical classifiers which are Minimum Distance Classifier, Bayes Minimum Distance Classifier. Given all the Hybrid Technique used in previous studies found that the use of hybrid techniques have been successful from other techniques, So we on a proposal by using the Hybrid Technique (ANFIS), which has already been used in ECG signal classification, But we will use Hybrid Technique (ANFIS) With Independent Component Analysis (ICA) and power spectrum, to extract the Important Feature from the ECG signal. That has not been addressed in the literature.

This paper proposes a new method for the classification of the cardiac rhythms. Feature extraction using Independent Component Analysis (ICA) and power spectrum, together with the RR interval then serve as input feature vector, this feature were used as input of FFNN, FIS, and ANFIS.

\section{INDEPENDENT COMPONENT ANALYSIS (ICA)}

ICA is a statistical and computational technique for revealing hidden factors that underlie sets of random variables, measurements, or signals. and it's a method for finding underlying factors or components from multivariate (multidimensional) statistical data. and has been successfully applied to numerous signal processing problems in areas as biomedicine, communications, finance, and remote sensing $[11,12]$. In ICA, the observed data are typically expressed as a linear combination of independent latent variables such that:

$$
\mathrm{v}=\mathrm{A} \mathrm{s}
$$

where $\mathrm{v}=[\mathrm{v} 1, \mathrm{v} 2, \ldots, \mathrm{vn}]$ is the vector of observed random variables, $\mathrm{s}=$ $[\mathrm{s} 1, \mathrm{~s} 2, \ldots, \mathrm{sn}]$ is the vector of statistically independent variables called the independent components, and $\mathrm{A}$ is the unknown mixing matrix. If we denote the columns of matrix A by aj the model can be written as:

$$
\mathrm{X}=\sum_{i=1} \mathrm{a}_{i} \mathrm{~s}_{i}
$$

In this model the only vector known is $\mathrm{x}$ and both $\mathrm{A}$ and $\mathrm{S}$ are estimated. 


\subsection{The Algorithm of ICA}

There are several ICA algorithms in use. Such as Fast-ICA algorithm, it developed by [13], also called a fast-fixed point algorithm.

\subsection{Fast Fixed-Point Algorithm}

The Fast-ICA algorithm [14] is a computationally highly efficient method for performing the estimation of ICA. It uses a fixed-point iteration scheme that has been found in independent experiments to be 10-100 times faster than conventional methods for ICA. Another advantage of the Fast-ICA algorithm is that it can be used to perform projection pursuit as well, thus providing a general-purpose data analysis method that can be used both in an exploratory fashion and for estimation of independent components (or sources).

\section{ADAPTIVE NEURO-FUZZY INFERENCE SYSTEM (ANFIS)}

ANFIS is one of hybrid Neuro-Fuzzy Inference Expert Systems and it works in Takagi-Sugeno-type fuzzy inference system, which was developed by Jang [13]. ANFIS is a Fuzzy Inference System formulated as a Feed-Forward Neural Network. Hence, the advantages of a fuzzy system can be combined with a learning algorithm.

\subsection{ANFIS Structure}

ANFIS architecture consists of five layers of nodes. out of the five layers, the first and the fourth layers consist of adaptive nodes there are Fuzzification and Defuzzification, while the second, third and fifth layers consist of fixed nodes there are Rule, Normalization, and Summation neuron. The adaptive nodes are associated with their respective parameters, get duly updated with each subsequent iterations while the fixed nodes are devoid of any parameters. To present the ANFIS architecture, two fuzzy ifthen rules based on a first order Sugeno model are considered: $[14,15,16]$.

Rule 1: If ( $\mathrm{x}$ is $\mathrm{A} 1)$ and $(\mathrm{y}$ is $\mathrm{B} 1)$ then $(\mathrm{fl}=\mathrm{p} 1 \mathrm{x}+\mathrm{q} 1 \mathrm{y}+\mathrm{r} 1)$

Rule 2: If ( $x$ is A2) and ( $y$ is B2) then ( $f 2=p 2 x+q 2 y+r 2)$

where $\mathrm{X}$ and $\mathrm{Y}$ are predefined membership functions, $\mathrm{Ai}$ and $\mathrm{Bi}$ are membership values, fi are the outputs and pi, qi, and ri are the consequent parameters that are updated in the forward pass in the learning algorithm. The ANFIS architecture to implement these two rules is shown in Figure.1, in which a circle indicates a fixed node, whereas a square indicates an adaptive node.

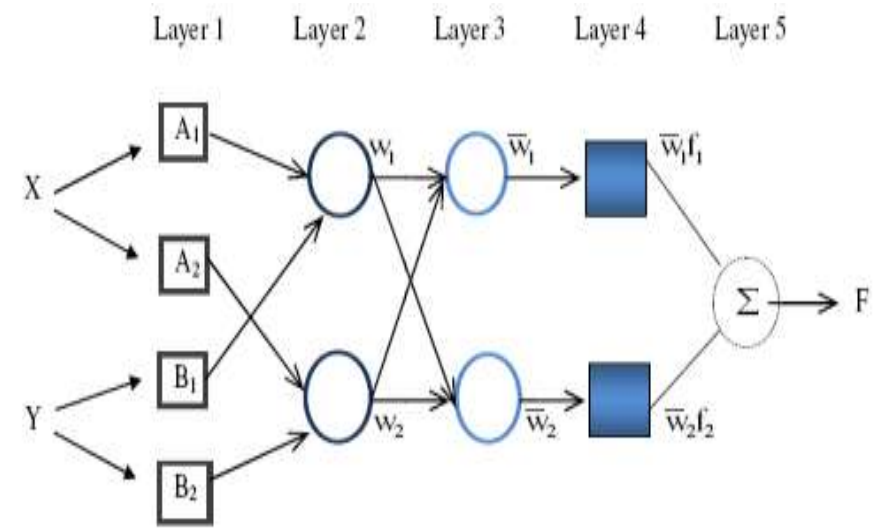

Figure.1 ANFIS architecture
International Journal of Computer Applications (0975 - 8887)

Volume 2 - No.4, June 2010

Layer 1: Fuzzification layer Every node I in the layer 1 is an adaptive node. The outputs of layer 1 are the fuzzy membership grade of the inputs, which are given by:

$$
\begin{aligned}
& \mathrm{O}_{\mathrm{i}}{ }^{1}=\mu_{\mathrm{A} i}(x), \quad \text { For } \mathrm{i}=1,2 \\
& \mathrm{O}_{\mathrm{i}}{ }^{1}=\mu_{\mathrm{B} i-2}(y), \text { For } \mathrm{i}=3,4
\end{aligned}
$$

where $\mathrm{x}$ and $\mathrm{y}$ is the inputs to node $\mathrm{i}$, where $\mathrm{A}$ is a linguistic label (small, large) and where $\mu \mathrm{Ai}(\mathrm{x}), \mu \mathrm{Bi}-2$ (y) can adopt any fuzzy membership function.

Usually we choose $\mu \mathrm{Ai}(\mathrm{x})$ to be bell-shaped with maximum equal to 1 and minimum equal to 0 , such as:

$$
\mu_{\mathrm{A} i}(x)=1 / 1+\left\{\left(x-c_{i} / a_{i}\right)^{2}\right\}^{\mathrm{b} \square}
$$

where (ai, bi and ci) are the parameters of the membership function. Parameters are referred to as premise parameters.

Layer 2: Rule layer a fixed node whose output is the product of all the incoming signals, The outputs of this layer can be represented as:

$$
\mathrm{O}_{\mathrm{i}}^{2}=w_{i}=\mu_{\mathrm{A} i}(x) \mu_{\mathrm{Bi}}(y) \quad i=1,2
$$

Layer 3: Normalization layer are also fixed node is a circle node.

$$
\mathrm{O}_{\mathrm{i}}^{3}=\square w_{i}=w_{i} /\left(w_{1}+w_{2}\right) \quad i=1,2
$$

Layer 4: Defuzzification layer an adaptive node with a node the output of each node in this layer is simply the product of the normalized firing strength and a first order polynomial.

$$
\mathrm{O}_{\mathrm{i}}{ }^{4}=\square w_{i} f_{i}=w_{i}\left(\mathrm{p}_{i} x+\mathrm{q}_{i} \mathrm{y}+\mathrm{r}_{i}\right) \quad i=1,2
$$

Layer5: Summation neuron a fixed node which computes the overall output as the summation of all incoming signals.

$$
\mathrm{O}_{\mathrm{i}}^{5}=\sum^{2} \square w_{i} f_{i=\sum_{i=1}^{2}} w_{i} f_{i} /\left(w_{1}+w_{2}\right)
$$

\subsection{Hybrid Learning of an ANFIS}

It is a Hybrid Neuro-Fuzzy technique that brings learning capabilities of Neural Networks to Fuzzy Inference Systems. The learning algorithm tunes the membership functions of a Sugeno-type Fuzzy Inference System using the training input-output data, using a back-propagation algorithm in combination with a least squares type of method.

\section{Data Description}

In this work we used lead-II ECG signals were obtained from the MIT-BIH arrhythmia database. We extracted from the MIT-BlH database in the same signal windows. The signal window length can be arbitrarily chosen provided that it is less than $10 \mathrm{sec}$. This is to satisfy the ANSI/AAMI EC13-1992 standard, which requires alarms for abnormal ECG signals to be activated within 10 seconds of their onset. All samples at a sampling frequency of $360 \mathrm{~Hz}$ in classes NSR, PVC, APC, from the other way at a 
sampling frequency of $250 \mathrm{~Hz}$ in classes VT, VF, and at a sampling frequency of $128 \mathrm{~Hz}$ in classes SVT.

A total of 420 datasets for six classes, out of which 280 datasets were used for training, and 140 datasets used for testing as show in table 1.

Table 1: datasets for six classes

\begin{tabular}{|l|c|c|c|}
\hline \multicolumn{1}{|c|}{ CLASSES } & Train set & Test set & Total \\
\hline Normal Sinus Rhythm (NSR) & 50 & 25 & 75 \\
\hline Premature Ventricular Contraction (PVC) & 50 & 25 & 75 \\
\hline Atrial Premature Contraction (APC) & 50 & 25 & 75 \\
\hline Ventricular Tachycardia (VT) & 50 & 25 & 75 \\
\hline Ventricular Fibrillation (VF) & 50 & 25 & 75 \\
\hline Supraventricular Tachycardia (SVT) & 30 & 15 & 45 \\
\hline
\end{tabular}

\section{Proposed Methods}

The block diagram of the proposed method for ECG beat classification is depicted in Figure.2. The method is divided into three steps: (1) preprocessing (2) calculation of feature vector and (3) classification by FFNN, FIS and ANFIS.

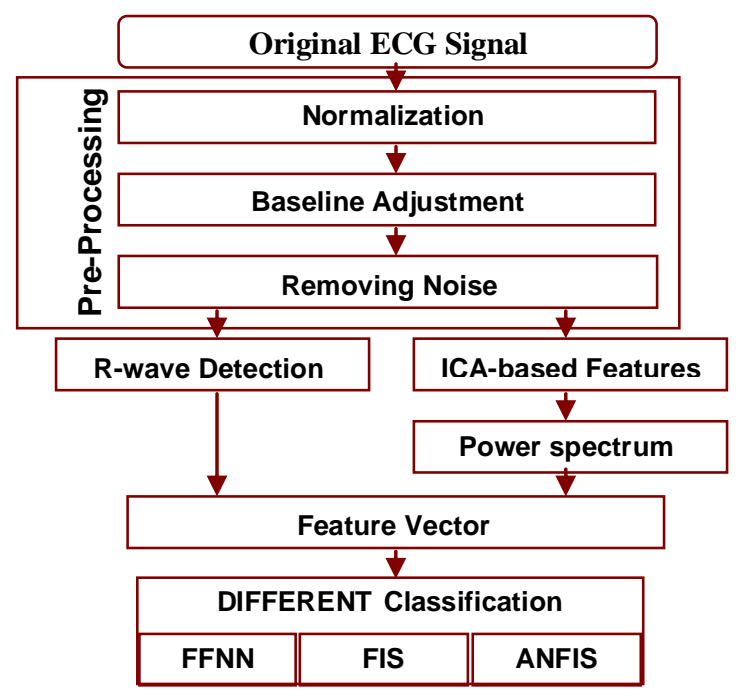

Figure.2 Block diagram of the proposed scheme for ECG beat classification.

\section{Pre-processing}

Each sample is pre processed by normalization process which necessary to standardize all the features to the same level [17]. After that the signal baseline may be shifted from zero line due to unexpected conditions such as patient movements, so that the baseline of the ECG signal was adjusted at zero line by subtracting the median of the ECG signal [18]. ECG signals can be contaminated with several types of noise, so we want to filter the signal. The unwanted noise of the signal must be removing. ECG were filtered using Low pass filter, high pass filter and Notch filter [19].

\subsection{Calculation of Feature Vector}

There are several algorithms for performing ICA. In this paper, a fast fixedpoint algorithm was used to estimate the independent components are show in Figure.3. [13,14]. The ICA features are then built into the Power Spectral to extract important feature, together with the RR interval then serve as input feature vector. There are five power spectrum features were extracted from the ECG signal at $(4,8,12,16$ and $20 \mathrm{~Hz})$ is show in
International Journal of Computer Applications (0975 - 8887)

Volume 2 - No.4, June 2010

Figure.4. The term power spectrum means the amount of power per unit (density) of frequency (spectral) as a function of the frequency [20].

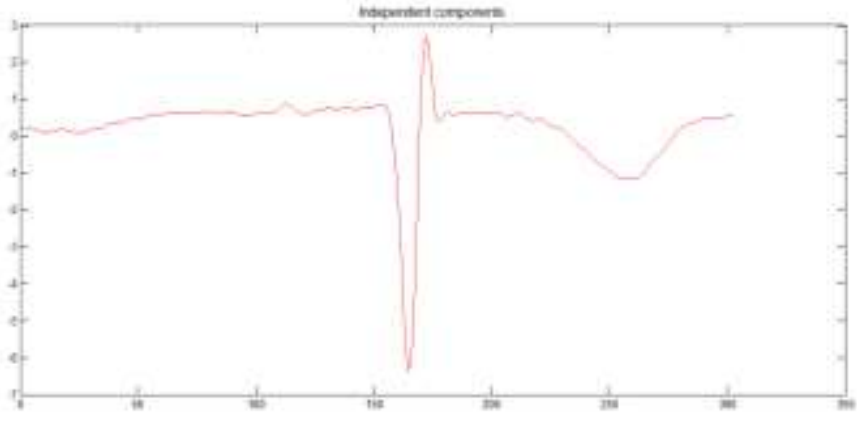

Figure.3 Independent Components

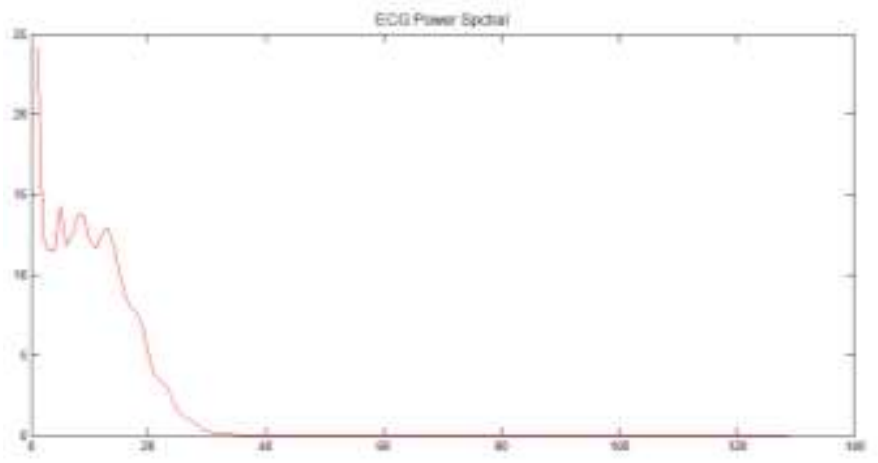

Figure. 4 power spectrum features were extracted from the ECG signal.

The RR interval between successive QRS peaks is considered as another important feature for recognizing many ECG arrhythmias. The RR interval is calculated as the time difference between the $\mathrm{R}$ points of the present and previous beat. There are several algorithms to Detect R-wave, we used PanTompkins algorithm [21].

\subsection{Classification Using Neural Network}

Neural Network is one of the most used methods of ECG beat classification, Multi-Layer Perception (MLP) based on the Neural Networks has been chosen to be able to classify the ECG signals, they are trained with Supervision, using Back- Propagation which minimize the squared error between the actual outputs of the network and the desired outputs.

Neural network structure consists of four layers (an input layer, two hidden layers, and output layer). using Feed-Forward Back-propagation, 7 neurons in the input layer, 12 neurons in the hidden layer, and 6 neurons in the output layer.

\subsubsection{Transfer Function Type and Number of Epochs}

Tan-sigmoid transfer function tansig is used in the input layer, hidden layers and the linear transfer function purelin is used in the output layer. The characteristics of tansig and purelin .

\subsection{Classification Using FIS}

Fuzzy classifiers are one application of Fuzzy theory. Expert knowledge is used and can be expressed in a very natural way using Linguistic Variables, which are described by Fuzzy sets.

The Sugeno Fuzzy method is used. Fuzzy logic if-then rules are formed by applying Fuzzy operations to these Membership Functions for given inputs. 
The Gaussian Membership Function is used for the input parameters. The range of the input parameters is normalized from 0 to 1 .

\subsection{Classification Using ANFIS}

An ANFIS based classifier is presented as a diagnostic tool to aid physicians in the classification of heart diseases. ANFIS using a strategy of hybrid approach of Adaptive Neuro-Fuzzy Inference System, we compose these two intelligent approaches, it will be achieve good reasoning in quality and quantity. In other words we have Fuzzy reasoning and network calculation. The objective of classification is to classifier six types of ECG signals, the feature vectors were applied as the input to an ANFIS classifier. The ANFIS converges with a smaller number of iteration steps with this hybrid learning algorithm. The ANFIS network has a total of 128 fuzzy rules and one output, the proposes ANFIS structure is show in figure 5 [22].

Table2 show Testing results of the ANFIS

\begin{tabular}{|c|c|c|c|c|c|c|c|}
\hline Classes & Testing Set & NSR & PVC & APC & VF & VT & SV \\
\hline NSR & 25 & 25 & 0 & 0 & 0 & 0 & 0 \\
\hline PVC & 25 & 0 & 25 & 0 & 0 & 0 & 0 \\
\hline APC & 25 & 0 & 0 & 25 & 0 & 0 & 0 \\
\hline VF & 25 & 0 & 0 & 0 & 23 & 2 & 0 \\
\hline VT & 25 & 0 & 0 & 0 & 1 & 24 & 0 \\
\hline SV & 15 & 0 & 0 & 0 & 0 & 1 & 14 \\
\hline
\end{tabular}

\subsubsection{Membership Function Type and Number of Epochs}

Gbell MFs are preferred by ANFIS in most cases. For other types of MFs preferred by the user for a certain application there is no rule in choosing them. The general rule is to obtain the best smallest error measure with minimum training parameters. The number of epochs is determined according to the accepted error measure, fixed by the user. In the present study 10 epochs have been taken.

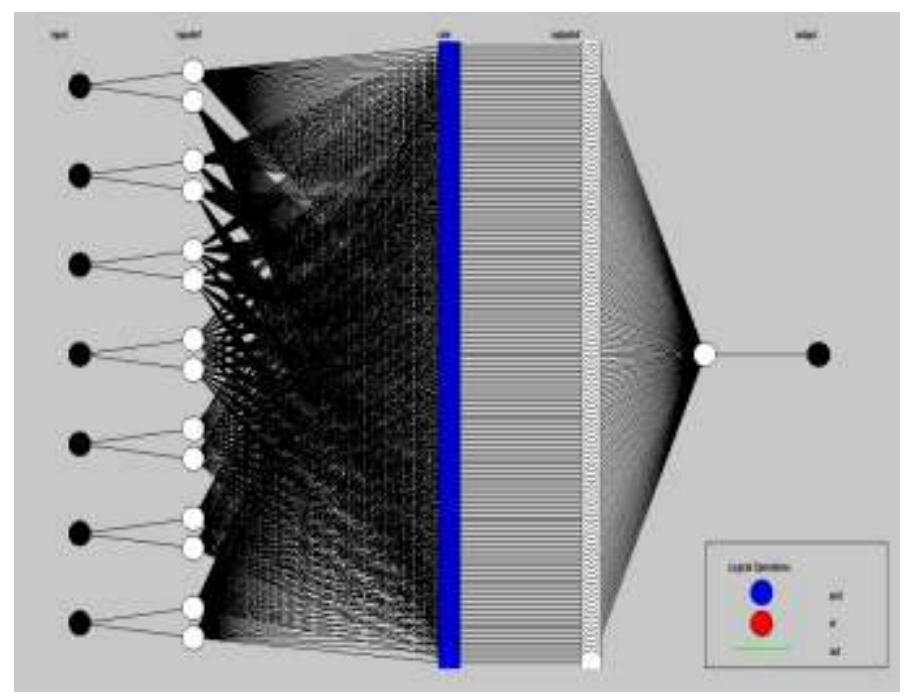

Figure.5 Proposes ANFIS structure

\section{Results and Discussion}

We get our bet classifier on the MIT-BIH Arrhythmia database. And we do not intend to detect the R-waves but use the information of R-waves provided in the MIT-BIH database which were manually verified by specialists. The Feature Vector that is obtained from the power spectrum
International Journal of Computer Applications (0975 - 8887)

Volume 2 - No.4, June 2010

and ICA Matrix is made up of $420 * 7$, which means the number of samples and five power spectrum features were extracted from the ECG signal at $(4,8,12,16$ and $20 \mathrm{~Hz})$ and two feature represented as follows The RR interval before the bet diseased and the RR interval after the bet diseased. The six types of ECG signals they are Normal Sinus Rhythm (NSR), Premature Ventricular Contraction (PVC), Atrial Premature Contraction (APC), Ventricular Tachycardia (VT), Ventricular Fibrillation (VF) and Supraventricular Tachycardia (SVT). The Training by using different methods were on a high level of efficacy $100 \%$. The classification by different methods were performed using MATLAB.

\section{Testing results of the ANFIS is show in Table2}

Two Ventricular Fibrillation was classified incorrectly as Ventricular Tachycardia. One Ventricular Tachycardia was classified incorrectly as Ventricular Fibrillation, and one Supraventricular Tachycardia was classified incorrectly as Ventricular Tachycardia. After training association rules in the form of IF then rules are generated and extracted. an example of initial and final (after rule extraction) decision surfaces are given in Fig.6.

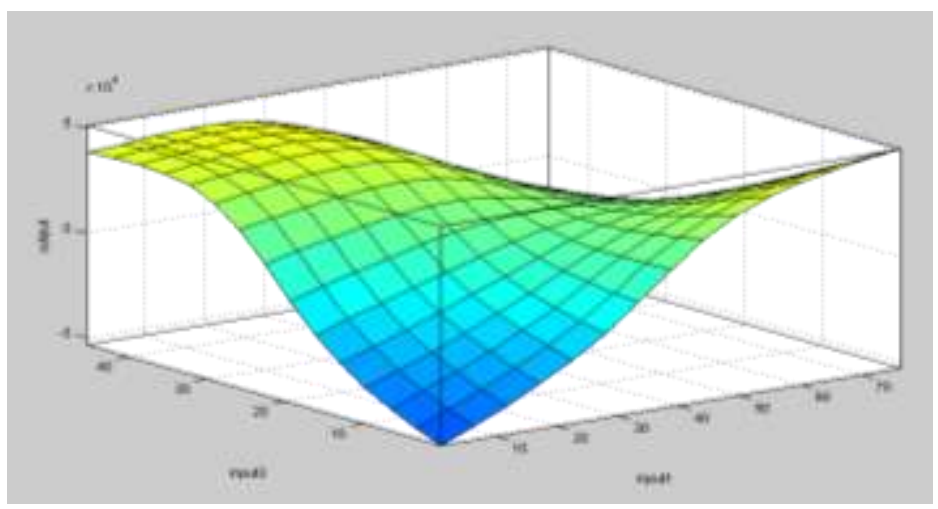

Figure 6.Final decision surface for input 1 and input 2

\section{Testing results of the FFNN is show in Table3}

Two normal sinus rhythms were classified incorrectly as Unknown. One premature ventricular contraction was classified incorrectly as Ventricular Fibrillation. One Ventricular Fibrillation was classified incorrectly as premature ventricular contraction and other one was classified incorrectly as Unknown. Three Ventricular Tachycardia was classified incorrectly as Unknown.

Table3 show Testing results of the FFNN

\begin{tabular}{|c|c|c|c|c|c|c|c|c|}
\hline Classes & $\begin{array}{c}\text { Testing } \\
\text { Set }\end{array}$ & NSR & PVC & APC & VF & VT & SV & Unk \\
\hline NSR & 25 & 25 & 0 & 0 & 0 & 0 & 0 & 0 \\
\hline PVC & 25 & 0 & 25 & 0 & 0 & 0 & 0 & 0 \\
\hline APC & 25 & 0 & 0 & 25 & 0 & 0 & 0 & 0 \\
\hline VF & 25 & 0 & 0 & 0 & 22 & 2 & 0 & 1 \\
\hline VT & 25 & 1 & 1 & 0 & 0 & 22 & 0 & 1 \\
\hline SV & 15 & 0 & 0 & 0 & 0 & 0 & 15 & 0 \\
\hline
\end{tabular}

Testing results of the FIS is show in Table4 
Two Ventricular Fibrillation was classified incorrectly as Ventricular Tachycardia and other one was classified incorrectly as Unknown. three Ventricular Tachycardia was classified incorrectly one was classified as normal sinus rhythm, the other one was classified as premature ventricular contraction and the other one was classified as Unknown. an example of initial and final (after rule extraction) decision surfaces are given in Fig.10.

Table4 show Testing results of the FIS

\begin{tabular}{|c|c|c|c|c|c|c|c|c|}
\hline Classes & $\begin{array}{c}\text { Testing } \\
\text { Set }\end{array}$ & NSR & PVC & APC & VF & VT & SV & Unk \\
\hline NSR & 25 & 23 & 0 & 0 & 0 & 0 & 0 & 2 \\
\hline PVC & 25 & 0 & 24 & 0 & 1 & 0 & 0 & 0 \\
\hline APC & 25 & 0 & 0 & 25 & 0 & 0 & 0 & 0 \\
\hline VF & 25 & 0 & 1 & 0 & 23 & 0 & 0 & 1 \\
\hline VT & 25 & 0 & 0 & 0 & 0 & 22 & 0 & 3 \\
\hline SV & 15 & 0 & 0 & 0 & 0 & 0 & 15 & 0 \\
\hline
\end{tabular}

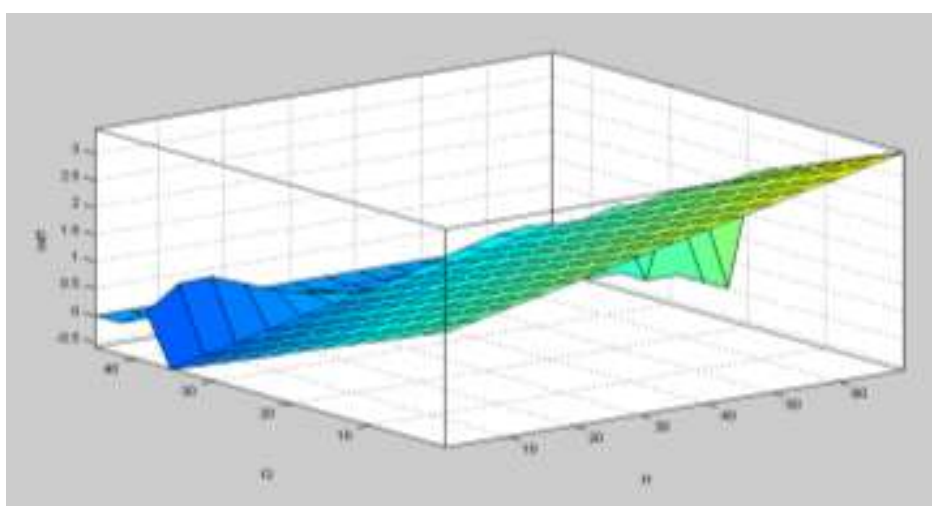

Figure 7.Final decision surface for input (f1) and output (f2)

Table5 summarizes the comparative results of these methods, in which the first row of the table is the result of the method ICA, Power Spectral, ANFIS classifier proposed in this paper. Among the nine methods, the proposed method outperforms the other methods with an impressive accuracy of $97.1 \%$ to discriminate six ECG beat types. Although this comparison may not be completely fair because of the different numbers and types of ECG beats tested, the propose scheme reveals to be a powerful tool in the computer-aided diagnosis of heart diseases based on ECG.

Table5 Comparative results of different methods

\begin{tabular}{|c|c|c|c|}
\hline Reference & Method & Number of beat types & Accuracy(\%) \\
\hline $\begin{array}{r}\text { Proposed } \\
\text { Method 1 }\end{array}$ & ANFIS-ICA & 6 & $97.1 \%$ \\
\hline $\begin{array}{r}\text { Proposed } \\
\text { Method 2 }\end{array}$ & FIS-ICA & 6 & $95.7 \%$ \\
\hline $\begin{array}{c}\text { Proposed } \\
\text { Method 3 }\end{array}$ & FFNN-ICA & 6 & $94.3 \%$ \\
\hline$[9]$ & ANFIS & 4 & $96.39 \%$ \\
\hline$[6]$ & FNN & 6 & $96 \%$ \\
\hline$[7]$ & ANFIS & 8 & $94 \%$ \\
\hline$[8]$ & ANFIS-DWT & 8 & $94 \%$ \\
\hline$[5]$ & Fuzzy-DWT & 8 & $93.13 \%$ \\
\hline$[10]$ & FFT-PCA-AR & 3 & 92.7 \\
\hline$[4]$ & BSS-Fourier & 5 & $85.04 \%$ \\
\hline
\end{tabular}

\section{Conclusion}

We propose a method that uses Hybrid approach of Adaptive Neuro-Fuzzy Inference System (ANFIS) model for classification of Electrocardiogram (ECG) signals. The hybrid classifier is computationally fast and classification achieved is better than the other two classifiers. ANFIS classifier demonstrated high classification accuracies and combines the benefits of both neural networks and Fuzzy Inference System, we have using combine independent component analysis (ICA), and power spectrum to extract important feature, together with the RR interval, then serve as input feature vector, this feature were used as input of FFNN, FIS, and ANFIS classifiers. six types of ECG samples were selected from the MIT-BIH arrhythmia database for experiments. The results indicate a high level of efficient, the proposed method outperforms the other methods with an impressive accuracy of $97.1 \%$, As for other methods FFNN, FIS results were respectively $94.3 \%, 95.7 \%$. In conclusion, our system has many advantages including efficiency, accuracy, and simplicity. We believe that it is very suitable to arrhythmic detection in clinical practice.

\section{Acknowledgements}

I would like to thank for the research the Faculty of Computer \& Information sciences, Department of Computer Science, Ain Shams University, who give financial support gratefully acknowledged.

\section{References}

[1] V.S. Chouhan and S.S. Mehta., "Detection of QRS Complexes in 12-lead ECG using Adaptive Quantized Threshold," International Journal of Computer Science and Network Security, January 2008.

[2] C. Wena, K. Chiung, T. Chiao and C. Hung, "Classification of ECG complexes using self-organizing CMAC," published by Elsevier Science Ltd, Taiwan, 2008.

[3] C. Chien, T. Hong and B. Yiliau, "Using Correlation Coefficient ECG Waveform For Arrhythmia Detection," Biomedical Engineering-Applications, Basis \& Communications, Feng Chia University, Taiwan, 2005.

[4] M. I. Owis, A. B. M. Youssef and Y. M. Kadah. "Characterisation of electrocardiogram signals based on blind source separation" ,Medical \& Biological Engineering \& Computing, Cairo, Egypt 2002.

[5] B.Anuradha,V.Reddy,"Cardiac Arhythmia Classification Using Fuzzy Classifiers", Journal of Theoretical and Applied Information Technology, 2008.

[6] Stanislaw Osowski and Tran Hoai Linh,“ ECG Beat Recognition Using Fuzzy Hybrid Neural Network”, IEEE Transactions on Biomedical Engineering, 2001.

[7] N. Kannathal, C.M. Lima, U. Rajendra Acharya and P.K. Sadasivan,"Cardiac state diagnosis using adaptive neuro-fuzzy technique”, Medical Engineering \& Physics, Singapore, 2006.

[8] B. Anuradha, K. Suresh Kumar and V. C. Veera Reddy, "Classification of Cardiac Signals Using Time Domain Methods", ARPN Journal of Engineering and Applied Sciences, Tirupati, India, 2008.

[9] Elif Derya Ubeyli, "Adaptive neuro-fuzzy inference system for classification of ECG signals using Lyapunov exponents", computer methods and programs in biomedicine, Ankara, Turkey, 2008 . 
[10] M. Mansor ,"Electrocardiogram (ECG) Classification Using Artificial Neural Networks," Master thesis, Department of Biomedical Engineering, Faculty of Engineering, Helwan Universty, Cairo, Egypt, 2007.

[11] A. Hyvarinen, J. Karhunen and E. Oja , "Independent Component Analysis," Wiley, 2001.

[12] E. Bingham and A. Hyvarinen , "A fast fixed-point algorithm for independent component analysis of complex valued signals," International Journal of Neural Systems, Vol. 10, No. 1, 2000.

[13] Jyh-Shing and Roger Jang., "ANFIS: Adaptive-Network-Based Fuzzy Inference System," computer methods and programs in biomedicine, IEEE Transactions on Systems, University of California, 1993.

[14] Abdulkadir Sengur., "An expert system based on linear discriminant analysis and adaptive neuro-fuzzy inference system to diagnosis heart valve diseases," Expert Systems with Applications, 2008.

[15] G. Zhao, C. Peng and Xiting Wang., "Intelligent Control for AMT Based on Driver's Intention and ANFIS Decision-Making," World Congress on Intelligent Control and Automation, 2008.

[16] Anupam Das, J. Maiti and R.N. Banerjee., "Process control strategies for a steel making furnace using ANN with bayesian regularization and ANFIS," Expert Systems with Applications, 2009.
[17] Surehka Palreddy,"ECG BEATS DATABASE DESCRIPTION", $\mathrm{Ph}$. D. University of Wisconsin, 1996.

[18] Yoon, S. W., Shin, H. S., Min, S. D., and Lee, M., "Adaptive motion artifacts reduction algorithm for ECG signal in textile wearable sensor", IEICE Electronics Express, Medical Electronics and Information Lab., Yonsei University,Vol.4, No.10, 312-318, Korea, 2007.

[19] Mahesh S. Chavan , R. A. Agarwala , M. D. Uplane, "Interference reduction in ECG using digital FIR filters based on rectangular window" , Wseas Transactions on Signal Processing, Shivaji University Kolhapur, INDIA, Issue 5, Volume 4, May 2008.

[20] K. Ichiro Minami, "Real-Time Discrimination of Ventricular Tachyarrhythmia with Fourier-Transform Neural Network", IEEE Transaction on Biomedical Engineering, vol.46, No 2, February 1999.

[21] R. M. Rangayyan "Biomedical Signal Analysis: A Case-Study Approach", IEEE, Inc. Wiley, 2002.

[22] S. Alavandar, M. J. Nigam "Adaptive Neuro-Fuzzy Inference System based control of six DOF robot manipulator", Journal of Engineering Science and Technology Review, India, 2008. 